\title{
Perú: Políticas para cerrar la brecha de la balanza de conocimientos
}

\section{Peru: Economic Policies to Close the Gap in the Knowledge Balance}

\author{
Santiago Roca ${ }^{1}$
}

Recibido: 4 de diciembre, 2010

Aceptado: 31 de enero, 2011

\section{Resumen}

El presente artículo advierte el déficit de la balanza de conocimientos del Perú en relación con el superávit de la balanza comercial y la necesidad de transformar la estructura y los coeficientes tecnológicos de las exportaciones para cerrar la brecha en dicha balanza de conocimientos. Asimismo, se analizan las principales políticas económicas que pueden ayudar a alcanzar este objetivo, especialmente la política industrial y de innovación tecnológica, el repensar los derechos de propiedad intelectual, así como impulsar las fuerzas de propagación del sistema económico. Se considera necesario el buen manejo de los fundamentos macroeconómicos y la apertura al mundo, ambos encuadrados bajo una conducción estratégica que permita añadir valor y equilibrar apropiadamente los intereses externos frente aquellos internos y los que pertenecen a la nación.

Palabras claves: Desarrollo económico, cambio tecnológico, industrialización, recursos naturales, política industrial, economía internacional, política comercial, estudio comparado de países, especialización productiva.

\begin{abstract}
In this paper we show the balance of knowledge deficit in Peru compared to the surplus of its trade balance and the necessity to transform the structure and technological coefficients of exports to close this knowledge gap. We analyze major economic policies that could help in closing the gap, especially industrial and technological innovation policies, rethinking intellectual property rights and impelling the forces that propagate the economic system as a whole. "Openness" and the "good management of macroeconomic fundamentals" are necessary but not sufficient conditions to transform the economy, which for better performance need the strategic "will and wit" of adding value and a better balance between external and national interests.
\end{abstract}

Keywords: Economic development, technological change, industrialization, natural resources, industrial policy, international economics, trade policy, comparative studies of countries, productive specialization.

1. Profesor Principal, Escuela de Administración de Negocios para Graduados, y Director del Centro de Propiedad Intelectual, Competencia, Consumidor y Comercio, CEPIC, de la Universidad ESAN. Ph.D. en Economía, Cornell University.

<sroca@esan.edu.pe> 


\section{LA BRECHA DE LA BALANZA DE CONOCIMIENTOS}

Si bien la balanza comercial de bienes y servicios del Perú ha sido positiva a partir del año 2004, la balanza de conocimientos ha tendido a ser a lo largo de los años cada vez más deficitaria, como se observa en la Tabla 1. El saldo negativo en la balanza de conocimiento significa que el Perú exporta menos conocimiento del que importa, lo que muestra el desbalance existente en ciencia y tecnología en relación con los países con los cuáles comerciamos. Utilizando datos de la OECD para el caso de las importaciones y del Perú para el caso de las exportaciones, se observa que los gastos de investigación y desarrollo invertidos (por categorías de bienes) son mucho mayores en las importaciones que en las exportaciones.
La Tabla 2 muestra, por ejemplo, que las importaciones de bienes de mediana y alta tecnología tienen mas contenido de conocimientos (5.88\% y $27.37 \%)$ que nuestras exportaciones $(2.03 \%$ y $9.43 \%)$. Igual ocurre con el conocimiento incorporado en las materias primas y recursos naturales: $0.65 \%$ y $1.29 \%$ en las importaciones versus $0.22 \%$ y $0.45 \%$ en las exportaciones. Si a ello se le añade que el $88.6 \%$ de las exportaciones de productos primarios y bienes basados en recursos naturales, mientras que el $46.9 \%$ de las importaciones son bienes de mediana y alta tecnología (Tabla 3), los hechos quedan consumados: la brecha de la balanza de conocimiento de bienes entre lo que el Perú importa y exporta es significativa, conforme se vio en la Tabla 1 (Roca, 2011).

Tabla 1.

Perú: Bienes y Servicios (en millones de dólares)

\begin{tabular}{lrrrrrr}
\hline & 1990 & 1994 & 1998 & 2002 & 2005 & 2008 \\
\hline Balanza Comercial (Bienes) & 358 & -1075 & -2462 & 321 & 5286 & 3090 \\
Balanza Comercial (Servicios) & -365 & -470 & -657 & -994 & -737 & -1962 \\
Balanza Comercial de Bienes y Servicios & -7 & -1545 & -3119 & -673 & 4549 & 1128 \\
Balanza de Conocimiento (Bienes) & -143 & -289 & -464 & -368 & -634 & -1541 \\
Balanza de Conocimiento (Servicios) & -91 & -114 & -196 & -202 & -249 & -435 \\
Balanza de Conocimientos de Bienes y & -234 & -404 & -660 & -570 & -883 & -1976 \\
Servicios & & & & & & \\
\hline
\end{tabular}

Fuente: Elaboración propia (cfr. Roca, 2011).

Tabla 2.

Perú: Coeficiente Tecnológico de Exportaciones e Importaciones 2008

(en porcentajes de las exportaciones e importaciones en términos corrientes)

\begin{tabular}{lcccccc}
\cline { 2 - 6 } & PP & BRN & BT & MT & AT & OT \\
\hline Exportaciones & 0.22 & 0.45 & 0.38 & 2.03 & 9.43 & 2.50 \\
Importaciones & 0.65 & 1.29 & 1.09 & 5.88 & 27.37 & 7.50 \\
\hline
\end{tabular}

Fuente: Elaboración propia (cfr. Roca, 2011).

Leyenda: $\mathrm{PP}=$ Productos Primario; $\mathrm{BRM}=$ Bienes Industrializados basados en Recursos Naturales;

$\mathrm{BT}=$ Bienes Industrializados de Baja Tecnología; $\mathrm{MT}=$ Bienes Industrializados de Mediana Tecnología;

$\mathrm{AT}=$ Bienes Industrializados de Alta Tecnología; OT = Otros Bienes. 
Tabla 3.

Perú: Composición Tecnológica de las Exportaciones e Importaciones 2008

(en porcentajes de las exportaciones e importaciones en términos corrientes)

\begin{tabular}{lccccccc}
\cline { 2 - 7 } & PP & BRN & BT & MT & AT & OT & TOTAL \\
\hline Exportaciones & 41.6 & 47 & 8.5 & 2.3 & 0.3 & 0.4 & 100 \\
Importaciones & 22.0 & 15.7 & 11.9 & 35.8 & 11.1 & 3.5 & 100 \\
\hline
\end{tabular}

Fuente: Elaboración propia (cfr. Roca, 2011).

Leyenda: $\mathrm{PP}=$ Productos Primarios; BRM $=$ Bienes Industrializados basados en Recursos Naturales; BT $=$ Bienes Industrializados de Baja Tecnología; MT = Bienes Industrializados de Mediana Tecnología; AT = Bienes Industrializados de Alta Tecnología; OT = Otros Bienes.

\section{¿CÓMO CERRAR LA BRECHA DE CONOCIMIENTOS?}

Conceptualmente, la balanza de conocimientos se puede equilibrar aparejando la composición y/o los coeficientes tecnológicos de las exportaciones e importaciones. Para ello se requiere principalmente - por el lado de las exportaciones - de políticas industriales, de aprendizaje e insumisión de tecnología, de formas inteligentes de promover la propiedad intelectual y de mecanismos que creen valor, articulen y propaguen los nuevos conocimientos elevando la productividad de toda la economía ${ }^{1}$.

\section{Políticas Industriales, de Aprendizaje e Insumisión de Tecnología}

La mayoría de países desarrollados ha optado estratégicamente por ofrecer incentivos, establecer instituciones y orientar las acciones de los actores en el mercado para lograr sus objetivos. Los países parten de una dotación de factores determinada que les da ventajas comparativas, pero no se dedican a explotar las ventajas hasta agotarlas. Se dan cuenta de que partiendo de lo que tienen deben construir un portafolio de actividades que les permita progresar en forma sostenida. Las estrategias conllevan riesgos y se puede incurrir en equivocaciones; por ello se enmiendan rumbos y existen períodos en que se progresa más y en otros menos. Por otro lado,

1 Por el lado de las importaciones, las políticas son más complicadas y menos autónomas por lo que por motivos de espacio no serán analizadas en el presente trabajo. el mundo es dinámico y cambiante, de tal manera que lo que funciona en un período puede resultar lo opuesto en otro, pues aparecen nuevos actores que entran a competir o a ofrecer nuevos productos.

Dos ejemplos de lo antedicho son los Estados Unidos y Nueva Zelanda, dos países con abundantes recursos naturales (Tabla 4). Los Estados Unidos, aún en las últimas décadas, sigue disminuyendo la participación de las exportaciones primarias del $21.61 \%$ en 1979 al 9.96\% en el 2008, y aumenta ligeramente la participación de las exportaciones de mediana y alta tecnología, del $52.16 \%$ en 1979 al $59.01 \%$ en el 2008 . Nueva Zelanda, otro país rico en recursos naturales, ha disminuido sus exportaciones de materias primas del $58.58 \%$ en 1979 al $44.25 \%$ en el 2008 y ha aumentado las exportaciones de bienes de mediana y alta tecnología del $5.97 \%$ en 1979 al $13.27 \%$ en el 2008 .

Para seguir con el ejemplo, en los Estados Unidos se aplica activamente la política industrial invirtiendo millones de dólares en el reentrenamiento laboral de miles de trabajadores, pagándoles inclusive sus salarios mientras estudian. Luego, el gobierno ayuda a estos trabajadores a reincorporarse en nuevas actividades que generan mayor valor y productividad ${ }^{2}$.

2 Cuando Estados Unidos discutía internamente el efecto de la apertura de algunos mercados a raíz de los Tratados de Libre Comercio, los sindicatos negociaban la reconversión laboral de los sectores potencialmente más afectados en programas hasta de 3 años, de forma que los trabajadores laboraran al final en otros sectores, trabajando menos y ganando más. 
Tabla 4.

Estructura tecnológica exportaciones (en porcentajes)

\begin{tabular}{lrrrrrr}
\hline En base a valores corrientes & \multicolumn{3}{c}{ Estados Unidos } & \multicolumn{3}{c}{ Nueva Zelanda } \\
\hline & \multicolumn{1}{c}{1979} & 1987 & \multicolumn{1}{c}{2008} & 1979 & 1987 & \multicolumn{1}{c}{2008} \\
\cline { 2 - 7 } Productos Primarios & 21.61 & 13.22 & 9.96 & 58.58 & 55.99 & 44.25 \\
B.I basados en recursos naturales & 14.32 & 14.28 & 16.98 & 27.38 & 25.05 & 31.08 \\
B.I de baja tecnología & 6.91 & 6.04 & 8.40 & 7.67 & 7.92 & 6.11 \\
B.I de tecnología media & 33.61 & 31.49 & 34.53 & 4.65 & 6.19 & 9.04 \\
B.I de alta tecnología & 18.55 & 26.65 & 24.48 & 1.32 & 2.29 & 4.23 \\
Otras Transacciones & 5.00 & 8.33 & 5.66 & 0.40 & 2.56 & 5.29 \\
Total & 100.00 & 100.00 & 100.00 & 100.00 & 100.00 & 100.00 \\
\hline
\end{tabular}

Fuente: Elaboración propia utilizando la base de datos de COMTRADE de las Naciones Unidas.

China, con abundante en mano de obra, no parece estar dispuesta a terminar especializada en la producción de bienes y servicios intensivos en manufactura. Más bien ha reducido las exportaciones primarias y de bienes de baja tecnología del $27.56 \%$ y el $36.69 \%$ respectivamente en 1987 al $2.36 \%$ y $30.53 \%$ en el $2008^{3}$. Los países desarrollados en general están conscientemente innovando y añadiendo conocimientos a sus actividades económicas, dejan las actividades de menor valor en la búsqueda de aquellas de mayor productividad (Reinert, 2007; Thompsom, 1989; Van Zandem, 2009).

Japón desde comienzos y Corea desde mediados del siglo XX se apoyaron en una amplia gama de políticas industriales que ofrecían protección dinámica a la industria incipiente. Ambas naciones subsidiaban a las exportaciones, conducían planes quinquenales, instalaban capacidades y desarrollaban habilidades. También coordinaban la inversión e inclusive regulaban la libre entrada y salida del mercado impulsando el mejoramiento tecnológico (Johnson, 1982; Magaziner \& Hout, 1980; Chang, 1993; Lall, 2000).

Muchos de los países en vías de desarrollo, entre ellos el Perú, no han sido capaces de aplicar coherentemente una política industrial en forma sostenida (Roca

3 Estimado por el autor con la base en datos en términos corrientes de COMTRADE.
\& Simabuko 1998, 2004; Jiménez, 2000), ni de copiar, aprender o, en función a ellas, construir y desarrollar innovaciones y nuevas tecnologías (Kuramoto \& Torero, 2009). La literatura sobre ciencia y tecnología es amplia en reconocer que las fallas de mercado son uno de los primeros obstáculos para el desarrollo tecnológico. Los problemas de información y coordinación son los primeros que aparecen en el mercado. El mercado por sí solo no aglutina ni procesa la información, tampoco la distribuye entre los interesados. Esta situación se aúna a que no existe ningún instrumento que permita coordinar las potenciales necesidades con las probables capacidades para que estas se pongan a disposición del desarrollo de las tecnologías.

De otro lado, los procesos de innovación, inclusive de transferencia tecnológica, son la mayor parte de las veces procesos que requieren de la intervención de diversos especialistas que por aproximaciones sucesivas y de ensayo y error, van palmo a palmo encontrando nuevas maneras de hacer y articular las cosas. Esto requiere de un alto grado de cooperación y familiaridad en el trabajo en equipo y un grado mínimo de organización que permita la formación de grupos y unidades de emprendimiento tecnológico. Mientras el Perú no resuelva estas fallas de información, coordinación, cooperación y organización no podrá absorber o procurar procesos generalizados de innovación, insumisión, transferencia y desarrollo de tecnologías. 
Pero ello no basta. La mayoría de países desarrollados han creado un conjunto de arreglos institucionales que tienen en sus canales la innovación y la creación de valor como ejes de su especialización productiva. Se integran desde el mismo sistema educativo incluyendo a las instituciones de investigación tecnológica públicas, el sistema de financiamiento de los emprendimientos tecnológicos, los medios de difusión y propagación de las tecnologías, los sistemas de transferencia, absorción y aprendizaje del exterior, y la inversión en infraestructura, normalización e instrumentación tecnológica.

El estado juega un rol medular invirtiendo fuertemente en investigación y desarrollo y mejorando la institucionalidad inventiva. Al mismo tiempo ofrece incentivos monetarios, premios, recompensas y exoneraciones tributarias a las empresas e individuos, que sirven para hacer prender la actividad innovadora (Reinert, 2004).

El sistema de patentes, creado por el estado para proteger y posibilitar a los inventores (empresas o individuos) a vivir mejor a través de la extracción de rentas extraordinarias a los consumidores, es un complemento y no un sustituto de todo lo anterior. Las patentes no resuelven las fallas del mercado y los problemas institucionales del mercado tecnológico. Estos deben ser enfrentados por decisiones estratégicas de todos los actores; no obstante, ellas refuerzan y benefician a las empresas cuyos países ya han resuelto estos problemas.

De otro lado, la habilidad ingeniera y tecnológica es diferente a la capacidad gerencial para lograr que las innovaciones puedan ponerse en práctica y ser exitosas comercialmente. Esto origina también que el sistema institucional de ciencia y tecnología busque engarzarse en el sistema productivo y empresarial y existan los suficientes medios y capacidades para valorizar y gestionar las nuevas tecnologías en los nuevos negocios. El sistema institucional también debe proveer para incentivarlos.

A ello se debe añadir los grandes cambios y oportunidades que se están originando por la revolución de las tecnologías de información a nivel mundial. Según Pérez (2010), se están abriendo ventanas de oportunidad originadas por la híper-segmentación de los mercados, las redes de valor global y la especialización tecnológica que permiten la producción especializada en pequeña escala y hasta personalizada, congruente con la dotación de factores y de organización empresarial y de recursos que tienen algunos países en desarrollo. La industria del turismo, artesanía, folclore y muchas de las industrias culturales entran en esta categorización. A ellas se añaden otras vinculadas a las plantas, a la biodiversidad y al procesamiento de los recursos naturales, tales como agroindustria, pesca-industria, minero-industria y turismo-industria.

La transformación de la estructura productiva y los coeficientes de conocimiento en las actividades económicas pasa por instaurar sistemas en donde todas las políticas públicas se evalúen y se revisen en relación con su potencial de contribuir a la generación, insumisión y difusión de conocimientos tecnológicos.

\section{Los Incentivos Morales, Sociales y Económicos: Los Derechos de Propiedad Intelectual}

La protección legal de los conocimientos a través del derecho de la propiedad intelectual se populariza en algunos países en el siglo XIX y XX en el supuesto que este es un instrumento para incentivar la actividad creativa y fomentar la innovación. Se protege a los inventores concediéndoles derechos monopólicos legales para la explotación comercial de sus inventos. Más que el incentivo a inventores individuales, lo que en realidad el derecho de propiedad intelectual ha impulsado es la creación de empresas y corporaciones que inviertan en la organización de emprendimientos tecnológicos a cambio de obtener los derechos monopólicos de las patentes. Los inventores en su mayoría son "empleados" de las corporaciones, quienes en algunos casos logran negociar y compartir algunos beneficios. Si bien inicialmente las corporaciones ejercían los derechos monopólicos solo en los territorios del país que instituía este incentivo, con el tiempo los países tecnológicamente mas avanzados se dieron cuenta de que ampliando el área de monopolización a otras regiones y países, podrían 
aumentar significativamente sus ganancias. Por eso es que desplegaron una ardua campaña mundial para que se ampliara la territorialidad a otros países, lo que se fue logrando en forma lenta y progresiva. Finalmente, en 1994 se aprobó el Tratado de Aspectos de Propiedad Intelectual Relacionado al Comercio, ADPIC, como parte del conjunto de normas que dieron nacimiento a la Organización Mundial de Comercio, $\mathrm{OMC}^{4}$.

Con este tratado, los países miembros de la OMC se comprometieron a instaurar un mínimo de reglas de propiedad intelectual obligatoria y vinculante para todos. Si bien los derechos de propiedad intelectual no se han llegado a internacionalizar, y cada país ejerce soberanía en su territorio, el ADPIC obliga a los países al trato nacional y de nación mas favorecida, lo que significa que nadie puede discriminar entre nacionales y extranjeros ni se puede favorecer a una nación más que a otra ${ }^{5}$. El marco está dado para que los tenedores de patentes puedan lograr que todos los consumidores del mundo paguen precios más altos por los objetos patentados. En el fondo, las patentes ya no son un instrumento por el que los consumidores nacionales consienten pagar precios monopólicos como estímulo a sus inventores para hacer que ellos y la patria progresen; en la práctica son los consumidores de todo el mundo los que están obligados a pagar precios extraordinarios a personas y corporaciones de otros países para que ellos sigan inventando y progresando.

Si la generación de propiedad intelectual fuera equitativa entre países, todos se beneficiarían del sistema y supuestamente las ganancias extraordinarias fomentarían la innovación en uno y otro país. Pero ¿qué incentivo recibe por ejemplo el Perú, si el $96 \%$ de las patentes que otorga pertenecen a extranjeros y no existe mayor actividad de invención tecnológica interna? ¿Sería el derecho de propiedad intelectual un estímulo, para fomentar verdaderamente la inventiva de los peruanos o más bien un instrumento para extraer rentas extraordinarias de nuestros consumidores en beneficio de las

4 Más información en $<$ http://www.wto.org/spanish/tratop_s/ trips_s/trips_s.htm>.

5 Excepto en casos determinados en la propia legislación. corporaciones de los países más avanzados? ¿Fomentan en verdad las patentes los inventos en el Perú?

Ni Europa ni los Estados Unidos (Machlup \& Penrose, 1950; Penrose, 1951) ni la mayoría de países que entraron al desarrollo después de ellos (Japón, Corea y hoy día China) han implementado en su momento de impulso el sistema de patentes. Más bien, ellos fomentaron la inventiva y la innovación atacando directamente las fallas de información, coordinación y cooperación inherentes a la actividad inventiva. El estado jugó un rol importante invirtiendo fuertemente en investigación y desarrollo y mejorando la institucionalidad inventiva, así como ofreciendo incentivos monetarios, premios y exoneraciones tributarias que hicieron prender la actividad innovadora (Bush 1957). Una vez que la inventiva despegó, empujaron el sistema de patentes para que las invenciones se sigan desarrollando. Así lo han hecho no solo Europa y los Estados Unidos, sino también Japón, Corea del Sur, Taiwán y Singapur,; por ese camino van también los países del BRICS: Brazil, Rusia, India, China y Sudafrica (Cassiolato \& Vitorino, 2009).

Por eso, en esta etapa de desarrollo del Perú, el estado, el sector académico y el sector privado no deben jugar al desarrollo de la extensión de los derechos de propiedad intelectual -interés actual de los países avanzados a través de los TLC's-, sino estimular los incentivos tributarios, financieros e institucionales e inclusive morales y sociales de los individuos y las empresas y organizaciones para que orienten sus recursos a la innovación tecnológica y la creatividad. Posteriormente, mejoradas las capacidades y habilidades iniciales se puede complementar estos instrumentos con el desarrollo de instrumentos de propiedad intelectual inteligentemente diseñados de acuerdo con las necesidades e intereses de los inventores y creadores nacionales.

Esto implica no copiar mecánicamente los acuerdos internacionales de propiedad intelectual, como ha sido la tendencia actual en el Perú, sino encontrar las variantes que hagan de los derechos de propiedad intelectual instrumentos más compatibles y consistentes con nuestro desarrollo. De esta manera se equilibra la cancha ofreciendo un terreno más parejo y equitativo 
con los otros países; así se ayuda a cerrar las brechas y se ofrece un mejor balance en la composición y coeficientes tecnológicos de los bienes, servicios y rentas que se producen y transan con el exterior ${ }^{6}$.

\section{Creación de Valor, Articulaciones y Fuerzas Propagadoras y Sistémicas}

Los sistemas productivos tendrán mayor impacto si son capaces de articular y generar sinergias, así como factores externos, que propulsen el progreso del sistema en su conjunto, entre ellos los clústers y los conglomerados. No se trata de aumentar solo la productividad micro-económica a través de innovaciones de procesos, productos y formas de gestión y organización, sino de articular vínculos y fuerzas propagadoras que difundan los nuevos conocimientos y eleven la productividad de todo el sistema.

Un ejemplo notable ocurre en el Perú en el sector gastronómico. Este sector, por sus características y alcance de oferta y demanda, no obtiene sus ventajas competitivas en la concentración de la oferta o los rendimientos a escala o en la protección individual de la propiedad intelectual. ¿De qué vale patentar los conocimientos culinarios cuando los mercados están hiper-fragmentados y nadie por sí solo será capaz de estar en todas partes a la vez? En este tipo de industria, la creación de valor y el progreso ocurren a través de procesos de difusión y propagación del conocimiento que enseñen a la mayor cantidad de productores a mejorar la calidad de sus servicios y a los consumidores a comer mejor.

En este caso no se trata de proteger sino de difundir, articular sub-sistemas y propagar la información de las mejores técnicas y prácticas entre miles de pequeños negocios. Con esto se creará valor y hará que todo el sector aumente sus coeficientes de conocimientos y prospere. Igual puede ocurrir en una multiplicidad de industrias que enfrentan mercados especializados, fragmentados o de nichos específicos, muchos de los cuáles

6 La Organización Mundial de Propiedad Intelectual, OMPI, tiene a partir del 2008 el mandato explícito de desarrollar un mejor balance de los instrumentos de propiedad intelectual en el crecimiento e intereses de los países en desarrollo. ocurren en las cadenas de valor global que existen en el mundo y que podrían aumentar significativamente los coeficientes de conocimiento incorporado de nuestras exportaciones. Por supuesto, no todos los sectores son iguales y cada uno de ellos tendrá que descubrir sus propias articulaciones y constelaciones sistémicas.

Las fuerzas de propagación que existen en la economía crecen cuando el sistema económico está bien organizado, interconectado y comunicado y su velocidad e intensidad multiplican sus efectos e impactos. Ello depende en realidad de cómo está organizado el sistema económico y de la necesidad de transformaciones estructurales para que las fuerzas propagadoras funcionen mejor. Es aquí donde el efecto sinérgico debe adquirir su mayor dimensión.

\section{CONDICIÓN NECESARIA PERO NO SUFICIENTE}

\section{La Necesidad de Coherencia y Consistencia en el Manejo de los Equilibrios Macroeconómicos Fundamentales}

Los esfuerzos para cambiar la estructura tecnológica de las actividades económicas requieren de la preservación de los equilibrios macroeconómicos fundamentales, tanto del sector fiscal, del sector externo y del gasto del sector privado. Si en el esfuerzo de producir mayores manufacturas para vender en el mercado interno se utilizan las materias primas que antes se exportaban, ello podría disminuir las entradas de moneda extranjera, desencadenando un problema de balanza de pagos y el estrangulamiento de la estrategia. $\mathrm{O}$, por el contrario, si el esfuerzo de industrialización es acompañado por una política de ingresos que eleva los sueldos y salarios para generar mercado a los productos manufacturados, sin que se haya previsto que ello pueda generar mayor déficit fiscal ó aumentar los requerimientos de insumos importados y de moneda extranjera, el proceso no podrá sostenerse y tendrá que revertir. De igual modo, si los esfuerzos de industrialización requieren invertir montos significativos en infraestructura básica para mejorar la competitividad y el estado o el sector privado enfrentan 
desequilibrios en sus finanzas y no se tiene manera de acceder a financiamiento externo, el esfuerzo de industrialización y de mejora de la productividad tampoco prosperará. La experiencia en el manejo de los equilibrios macroeconómicos para sostener los procesos de industrialización está ampliamente documentada en el estudio sobre los países del este asiático realizado por el Banco Mundial (1993).

La experiencia en el Perú en las décadas de los setentas y ochentas en cuanto a la falta de coherencia $\mathrm{y}$ consistencia en los equilibrios macroeconómicos sectoriales es también aleccionadora. Bajo políticas activas que promovieron la industrialización, el aumento de las actividades manufactureras en los períodos de 1970 a 1975, 1979 a 1981 y de 1985 a 1987 no pudieron sostenerse por desequilibrios externos o fiscales, y originaron la reversión del proceso y el regreso a los niveles previos de primarización (Roca \& Simabuko, 2004). En la década de los noventas, paradójicamente bajo una política neoliberal y sin ninguna conducción estratégica, los flujos internacionales de capital permitieron sostener el desequilibrio de la balanza comercial y reducir por sí solo, aunque marginalmente, la primarización (Roca \& Simabuko, 2004).

En la presente década, sin embargo, no se ha aprovechado la ocasión para empujar la industrialización y la mejora tecnológica de la estructura productiva, a pesar de los holgados resultados en las cuentas externas y fiscales. La economía ha estado dirigida por los intereses de grandes empresas extractivas y los designios del libre mercado. La experiencia es interesante ya que se necesita coherencia y consistencia en el manejo de los equilibrios macroeconómicos sectoriales para poder cambiar la estructura productiva y exportadora de la economía.

\section{OTROS INGREDIENTES}

\section{La Apertura no es Suficiente}

Algunos estudios enfatizan que la apertura y los tratados de libre comercio crean de por sí las condiciones para el mejoramiento tecnológico y el cambio en la especialización productiva (Balassa et al., 1986; Williamson, 1989). Esto, sin embargo, ha sido refutado por otros autores que argumentan que la apertura no es suficiente para lograr el cambio de la estructura productiva (Rodrik, 2005; Chang, 2002). Los países que han cambiado su estructura exportadora han tenido casi todos una agresiva política pro exportadora, pero ella ha sido generalmente acompañada por una política industrial y tecnológica de creación de valor y adición de conocimientos.

Una política pro exportadora no es sinónima de una zona de libre comercio. Los casos más notables son Corea y Japón: estos países promovieron sus exportaciones, pero principalmente fomentaron la creación de valor y el mejoramiento tecnológico de sus sistemas productivos locales (Ernst, 1998). En México, por lo contrario, la zona de libre comercio ha instalado una industria de ensamblaje que hasta hoy sigue extremadamente lenta en la generación de nuevo conocimiento (Fujii et al. 2005; Roca \& Simabuko, 2003). Por lo tanto, ni la apertura ni las zonas de libre comercio son suficientes por sí mismas para lograr aumentar la intensidad o los niveles de conocimiento local de las actividades económicas.

\section{Conducción Estratégica y Balance entre lo Interno y lo Externo}

Para cerrar la brecha de conocimientos no basta la apertura, la mirada hacia fuera y dejar que los mercados actúen por sí solos. Se necesita articular una estrategia que cambie gradualmente la especialización productiva y aumente el coeficiente de conocimientos incorporados en las actividades productivas, logrando su multiplicación y propagación al resto de las actividades económicas.

Cambiar la composición y los coeficientes tecnológicos de las exportaciones requiere cambios en la conducción estratégica. Ello permitirá obtener un balance entre lo interno y lo externo como ingredientes necesarios para mantener y construir ventajas competitivas que añadan valor, transformen el aparato productivo y lleven al crecimiento sostenido. Los países 
como Corea o Japón buscaron en su momento aprovechar las ventajas que ofrecía el mundo exterior, pero seleccionaron las actividades estratégicas y los grados de regulación que obligaron a los actores económicos externos e internos a actuar en mayor concordancia con los objetivos del país.

\section{CONCLUSIONES}

El déficit de conocimiento en los bienes y servicios que el Perú comercia con el exterior es muy significativo. Por más que la balanza comercial mantenga el superávit, la balanza de conocimientos es cada vez más deficitaria y el país está equivocadamente especializándose en actividades de poco valor. No solo existe una marcada diferencia en la composición tecnológica de las exportaciones e importaciones, sino que el contenido de conocimiento incorporado en cada categoría tecnológica es mucho mayor en las importaciones que en las exportaciones. Los coeficientes tecnológicos de los productos que se importan casi triplican a los que se exportan dentro de su misma categoría. Asimismo, los productos de alta tecnología tienen aproximadamente 42 veces mas conocimiento incorporado que los productos primarios, 21 veces más que los basados en recursos naturales, 25 veces más que los de baja tecnología, 4.65 veces más que los de mediana tecnología (inferido de las Tablas 2 y 3 ).

Lo antedicho significa que con los actuales coeficientes tecnológicos por tipos de bienes, el Perú se verá invariablemente perjudicado en el intercambio de conocimientos con el exterior. Esto significa que se requerirá cada vez mas unidades de bienes primarios para comprar una unidad de alta tecnología, ensanchándose las diferencias de horas de trabajo e ingreso con los productores de bienes de alta tecnología de los países con los cuáles comerciamos.

El Perú tiene, sin embargo, un amplio techo para incrementar el conocimiento incorporado y mejorar la especialización de los bienes que actualmente produce y exporta. Por ejemplo, se podría incrementar el conocimiento incorporado en los productos primarios y productos basados en recursos naturales del $0.22 \%$ y $0.45 \%$ respectivamente al $0.65 \%$ y $1.29 \%$ añadiendo nuevas tecnologías y mejorando la calidad y el valor agregado de esos productos. Igual incremento se podría incorporar a los productos de baja, mediana y alta tecnología. Concurrentemente, se podría ir cambiando de manera paulatina la composición de las exportaciones con una mejor visión y conducción estratégica nacional que dirija los recursos y obtenga un mejor balance y consistencia en la orientación hacia fuera y hacia adentro de la economía nacional.

La miopía que se observa actualmente en la especialización exportadora del país se debe los siguientes factores: 1) la abdicación deliberada de nuestros gobernantes de alcanzar las metas trazadas y de lograr una conducción estratégica que añada valor e intervenga para mejorar las capacidades de incorporar conocimientos y nuevas tecnologías; 2) aferrarse a la ilusión de que los recursos naturales son inagotables; 3) la creencia de que los intereses empresariales de corto plazo son necesariamente coincidentes con los fines de desarrollo y progreso de la nación de mediano y largo plazo; 4) la sujeción de las políticas públicas a los intereses y fines de las actividades extractivas, principalmente en manos de corporaciones y empresas del exterior.

Los esfuerzos por cambiar la composición tecnológica de las exportaciones enfrentan la necesidad de coherencia y consistencia en el manejo de los equilibrios macroeconómicos sectoriales. El Perú es un ejemplo de cómo los desequilibrios macro sectoriales hicieron retroceder y dejaron en peor situación la economía en los esfuerzos de industrialización de las décadas de los sesenta y setenta del siglo pasado.

Si se desea cerrar las brechas de conocimiento es necesario resolver las fallas de mercado y fortalecer un sistema institucional que incentive a todos los actores en el desarrollo tecnológico. Para ello no solamente se necesita fortalecer las capacidades productivas y fuerzas impulsoras del desarrollo tecnológico, sino también mejorar la organización y las fuerzas propagadoras de la economía para difundir y expandir los efectos sinérgicos y de expansión del sistema económico como un todo. 


\section{Referencias}

Balassa, B.: Bueno, G. M.; Kuczyinski, P. P. \& Simonsen, M. H. (1986): Toward Renewed Economic Growth in Latin América, Institute for International Economics, Washington D. C.

Bush, V. (1957). Some Proposals for Improving the Patent System. Journal of the Patent and Trademark Office Society, 39(1).

Cassiolato, J. E., \& Vitorino, V. (2009). BRICS and Development Alternatives: Innovation Systems and Policies. London and New York: Anthem Press.

Chang, H (1993). The Political Economy of Industrial Policy in Korea", Cambridge Journal of Economics, 17(2), 131-157.

Chang, H. (2002). Kicking Away the Ladder: Development Strategy in Historical Perspective. London: Anthem Press.

Ernst, D. (1998). Catching Up, Crisis and Industrial Upgrading: Evolutionary Aspects of Technological Learning in Korea's Electronic Industry. Asia Pacific Journal of Management, 15(2), 247-283.

Fujii G., Candaudap, E., \& Gaona, C. (2005). Exportaciones, Industria Maquiladora y Crecimiento Económico en México a partir de la década de los Noventa. Investigación Económica, 254, 125-156.

Kuramoto, J., \& Torero, M. (2009). Public-private research, development, and innovation in Peru. En M. Graham \& J. Woo Fueling Economic Growth: the Role of Public-Private Sector Research in Development. Ottawa, Canadá: IDRC,.

Jiménez, F. (2000). Liberalización, Reestructuración Productiva y Competitividad en la Industria Peruana de los Años 90 (Documento de Trabajo, no. 183). Pontificia Universidad Católica del Perú, Departamento de Economía.

Johnson, C. (1982). MITI and The Japonese Miracle. Stanford, MA: Stanford University Press.

Lall, S. (2000). The Technological Structure and Performance of Developing Country Manufactured
Exports, 1985-98. Oxford Development Studies, 28(3), 337-369.

Machlup, F., \& Penrose, E. (1950). The Patent Controversy in the Nineteenth Century. Journal of Economic History, 10(1), 1-29.

Magaziner, I., \& Hout, T. (1980). Japonese Industrial Policy. London: Policy Studies Institute.

OECD (2009). Base de Datos STAN Indicators. Recuperado de http://stats.oecd.org/index.aspx.

Penrose, E (1951). The Economics of the International Patent System. Baltimore: John Hopkins University Press.

Pérez, C. (2010). Dinamismo Tecnológico e Inclusión Social en América Latina: Una estrategia de Desarrollo productivo Basado en Recursos Naturales. Revista de la CEPAL, 100, 123-145.

Reinert, E. (2004). The Other Canon: the History of Renaissance Economics. En E. Reinert (Ed) Globalization, Economic Development and Inequality: An Alternative Perspective. Cheltenham, UK: Edward Elgar Publishing.

Reinert, E. (2007). How Rich Countries Got Rich and Why Poor Countries Stay Poor. London: Editorial Constable.

Roca, S. (Ed) (2007). Propiedad Intelectual y Comercio en el Perú: Impacto y Agenda Pendiente. Lima, Perú: ESAN Ediciones.

Roca, S. (2011). La Balanza de Conocimientos del Perú. En Perú al 2021, Retos y Perspectivas para el Empresario: Un análisis multidisciplinario. Buenos Aires, Argentina: CENGAGE Learning.

Roca, S. \& Simabuko, L. (1998). Primarización y Nivel de Vida: El Caso Peruano 1950-1997 (Documentos de Trabajo No 8). Universidad ESAN, ESAN Ediciones.

Roca, S., \& Simabuko, L. (2003). Little Value Creation, Articulation and Propagation Forces in The Mexican Economy. Paper presentado en la Segunda Conferencia Globelics, Rio de Janeiro, Brasil. 
Roca, S., \& Simabuko, L. (2004). Natural resources, industrialization and fluctuating standards of living in Peru, 1950-97: a case of activity-specific economic growth. En E. Reinert Globalization, Economic Development and Inequality. An Alternative Perspective. Cheltenham, UK: Edward Elgar Publishing.

Rodrik, D. (2005). Políticas de Diversificación Económica. Revista de la CEPAL 87(diciembre), 7-23.

Thompsom, G. (1989). Industrial Policy: USA and UK Debates. London: Routledge.
Van Zanden, L. (2009). The Long Road to the Industrial Revolution: The European Economy in a Global Perspective, 1000-1800. Leiden, The Netherlands Koninklijke Brill.

Williamson, J. (1989). What Washington D. C Means by Policy Reform. In Latin American Adjustment: How Much Has Happened?. Washington, D. C.: Institute of International Economics.

World Bank. (1993). The East Asian Miracle. Oxford, UK: Oxford University Press. 